\title{
RIGIDITY OF CR MAPS BETWEEN SHILOV BOUNDARIES OF BOUNDED SYMMETRIC DOMAINS
}

\author{
SUNG-YEON KIM* AND DMITRI ZAITSEV**
}

\begin{abstract}
Our goal is to establish what seems to be the first rigidity result for CR embeddings between Shilov boundaries of bounded symmetric domains of higher rank. The result states that any such CR embedding is the standard linear embedding up to CR automorphisms. Our basic assumption extends precisely the well-known optimal bound for the rank one case. There are no other restrictions on the ranks, in particular, the difficult case when the target rank is larger than the source rank is also allowed.
\end{abstract}

\section{INTRODUCTION}

Rigidity phenomena for holomorphic isometries into complex space forms go back to Bochner [Bo47] and Calabi [Ca53] and lead to far going and deeper understanding of metric rigidity between general bounded symmetric domains in the work of Mok, Mok-Ng and Ng among others. The reader is referred to the survey by Mok [M11] for more details, see also the very recent work by Yuan-Zhang [YZ10]. Other important rigidity phenomena for bounded symmetric domains, such as the strong rigidity of complex structures of their compact quotients have been discovered by Siu [S80, S81].

On the other hand, the study of rigidity of holomorphic maps originated in the work of Poincaré [P07] and later Alexander [A74] for maps sending one open piece of the sphere into another. It was Webster [W79] who first obtained rigidity for holomorphic maps between pieces of spheres of different dimension, proving that any such map between spheres in $\mathbb{C}^{n}$ and $\mathbb{C}^{n+1}$ is totally geodesic. Further results in this direction are due to Faran [Fa86], Cima-Suffridge [CS83, CS90], Forstneric [F86, F89] and Huang [H99] who obtained the best known regularity assumption independent of the dimension difference $n^{\prime}-n$, for CR maps between pieces of spheres in $\mathbb{C}^{n+1}$ and $\mathbb{C}^{n^{\prime}+1}$ under the assumption $n^{\prime}<2 n$. Beyond this bound, the rigidity is known to fail as illustrated by the so-called Whitney map (see e.g. Example 1.1 in [EHZ04]). (CR maps are closely related to holomorphic ones, see e.g. [BER99]). We mention the work by Huang-Ji [HJ01], Huang [H03] and Huang-Ji-Xu [HJX06] dealing with this more difficult case, where rigidity has to be replaced by the classification of the maps. On the other note, further rigidity phonemena for CR maps between real hypersurfaces and hyperquadrics have been discovered by Ebenfelt-Huang and the second author [EHZ04, EHZ05], Baouendi-Huang [BH05], Baouendi-Ebenfelt-Huang [BEH09] and Ebenfelt-Shroff [ES10].

2000 Mathematics Subject Classification. 32V40, 32V30, 32V20, 32M05, 53B25, 35N10.

Key words and phrases. bonded symmetric domains, symmetric CR manifold, CR embedding, complete system, totally geodesic embedding.

*This research was supported by Basic Science Research Program through the National Research Foundation of Korea(NRF) funded by the Ministry of Education, Science and Technology (grant number 2009-0067947).

**Supported in part by the Science Foundation Ireland grant 10/RFP/MTH2878. 
However, comparing with metric rigidity mentioned above, holomorphic rigidity for maps between bounded symmetric domains $D$ and $D^{\prime}$ of higher rank remains much less understood. If the rank $r^{\prime}$ of $D^{\prime}$ does not exceed the rank $r$ of $D$ and both ranks $r, r^{\prime} \geq 2$, the rigidity of proper holomorphic maps $f: D \rightarrow D^{\prime}$ was conjectured by Mok [M89] and proved by Tsai [Ts93], showing that $f$ is necessarily totally geodesic (with respect to the Bergmann metric).

The remaining case $r<r^{\prime}$ seems to be very hard and only little is known. Tu [Tu02a, Tu02b] established holomorphic rigidity respectively in the equidimensional case (when he proves that the map is biholomorphic and hence $r=r^{\prime}$ ) and for maps between Cartan type I bounded symmetric domain $D_{p, p-1}$ and $D_{p, p}$ (see below). Finally, Mok [M08] proved the nonexistence of proper holomorphic maps between certain pairs of bounded symmetric domains with arbitrary $r^{\prime}-r$.

The goal of this paper is to take on the rigidity problem for locally defined $C R$ embeddings between Shilov boundaries of general Cartan type I bounded symmetric domains $D_{p, q}$ of higher rank. This includes the interesting case $r<r^{\prime}$. To the best authors' knowledge all known results on local CR rigidity deal with maps between real hypersurfaces and rely heavily on Tanaka-Chern-Moser approach [Ta62, CM74] and many of them also on Tanaka-Webster connection, which is unavailable for Shilov boundaries of higher rank. In this paper we follow a new approach going back to the general Cartan's moving frame method. To compensate for the lack of the power of Tanaka-ChernMoser normalization, we introduce a sequence of several subsequent adjustments of moving frames reaching further and further normalization conditions. We prove:

Theorem 1.1. Let $f$ be a smooth $C R$ embedding between open pieces of Shilov boundaries of two bounded symmetric domains $D_{p, q}, D_{p^{\prime}, q^{\prime}}$ of Cartan type I with $q<p, q^{\prime}<p^{\prime}$. Assume that the rank $q>1$ and

$$
p^{\prime}-q^{\prime}<2(p-q) .
$$

Then after composing with suitable automorphisms of $D_{p, q}$ and $D_{p^{\prime}, q^{\prime}}, f$ is given by the block matrix

$$
z \mapsto\left(\begin{array}{cc}
z & 0 \\
0 & I \\
0 & 0
\end{array}\right) .
$$

Note that the assumptions $q<p$ and $q^{\prime}<p^{\prime}$ exclude precisely the cases of square matrices, where one of the Shilov boundaries is totally real and consequently CR maps are trivial. Furthermore, our basic assumption (1.1) corresponds precisely to the optimal bound $n^{\prime}<2 n$ mentioned before in the rank 1 case $\left(q=q^{\prime}=1\right)$ of maps between spheres, where $n=p-1$ and $n^{\prime}=p^{\prime}-1$ are the CR dimensions of the spheres.

Acknowledgement. The authors thank the anonymous referee for careful reading and helpful remarks.

\section{Preliminaries AND ADAPted FRAmeS}

Throughout this paper we adopt the Einstein summation convention unless mentioned otherwise. However, if two equal indices appear at the same letter, e.g. $\Phi_{a}{ }^{a}$, no summation is assumed. We shall also follow the convention that small Greek indices $\alpha, \beta, \gamma, \delta$ run over $\{1, \ldots, q\}$, small Latin indices 
$i, j, k, l$ over $\{1, \ldots, n\}$, small Latin indices $a, b, c, d$ over $\left\{1, \ldots, q^{\prime}\right\}$ and large Latin indices $I, J, K, L$ over $\left\{1, \ldots, n^{\prime}\right\}$.

Recall that $D_{p, q}$ has the standard realization in the space $\mathbb{C}^{p \times q}$ of $p \times q$ matrices, given by

$$
D_{p, q}:=\left\{z \in \mathbb{C}^{p \times q}: I_{q}-z^{*} z \text { is positive definite }\right\},
$$

where $I_{q}$ is the identity $q \times q$ matrix and $z^{*}=\bar{z}^{t}$. The Shilov boundary of $D_{p, q}$ is given by

$$
S_{p, q}=\left\{z \in \mathbb{C}^{p \times q}: I_{q}-z^{*} z=0\right\} .
$$

In particular, $S_{p, q}$ is a symmetric CR manifold of CR dimension $(p-q) \times q$ in the terminology of [KZ00]. For $q=1, S_{p, 1}$ is the unit sphere in $\mathbb{C}^{p}$. We shall always assume $p>q$ so that $S_{p, q}$ has positive CR dimension, i.e. not totally real.

Example 2.1. The following generalization of the well-known Whitney map

$$
\left(\begin{array}{ccc}
z_{11} & \cdots & z_{1 q} \\
\vdots & \ddots & \vdots \\
z_{p 1} & \cdots & z_{p q}
\end{array}\right) \mapsto\left(\begin{array}{cccccc}
z_{11} & \cdots & z_{1 q^{\prime}} & 0 & \cdots & 0 \\
\vdots & \ddots & \vdots & \vdots & \ddots & \vdots \\
z_{p-1,1} & \cdots & z_{p-1, q^{\prime}} & 0 & \cdots & 0 \\
z_{11} z_{p 1} & \cdots & z_{11} z_{p q^{\prime}} & 0 & \cdots & 0 \\
\vdots & \ddots & \vdots & \vdots & \ddots & \vdots \\
z_{p 1} z_{p 1} & \cdots & z_{p 1} z_{p q^{\prime}} & 0 & \cdots & 0 \\
0 & \cdots & 0 & 1 & \cdots & 0 \\
\vdots & \ddots & \vdots & \vdots & \ddots & \vdots \\
0 & \cdots & 0 & 0 & \cdots & 1
\end{array}\right) \in \mathbb{C}^{(p+m) \times\left(q^{\prime}+m\right)}
$$

restricts to a CR map between the Shilov boundaries, where $1 \leq q^{\prime} \leq q$ and $m$ is arbitrary. This map is not injective in $D_{p, q}$ and hence is not linear after composing with any automorphisms of $D_{p, q}$ and $D_{p^{\prime}, q^{\prime}}^{\prime}$. For $q=q^{\prime}=1, m=0$, this is the classical Whitney proper map between unit balls in $\mathbb{C}^{p}$ and $\mathbb{C}^{2 p-1}$ respectively, which corresponds to the equality in (1.1) showing that the latter is an optimal bound.

Example 2.2. The following examples show that there are lots of CR maps between Shilov boundaries for any choices of ranks $q$ and $q^{\prime}$. Fix a collection of proper maps $\varphi_{1}, \ldots, \varphi_{q^{\prime}}$ from the unit ball in $\mathbb{C}^{p}$ into unit balls in $\mathbb{C}^{m_{1}}, \ldots, \mathbb{C}^{m_{q^{\prime}}}$ respectively for any choice of integers $m_{1}, \ldots, m_{q^{\prime}}$. For any $q$, and any choice of integers $j_{1}, \ldots, j_{q^{\prime}} \in\{1, \ldots, q\}$, define

$$
\Phi: \mathbb{C}^{p \times q} \rightarrow \mathbb{C}^{\left(m_{1}+\ldots+m_{q^{\prime}}\right) \times q^{\prime}},
$$

such that $\Phi(Z)$ is the block-diagonal matrix with entries $\varphi_{1}\left(z_{j_{1}}\right), \ldots, \varphi_{n}\left(z_{j_{q^{\prime}}}\right)$ on the diagonal. Then $\Phi$ restricts to a $\mathrm{CR}$ map between Shilov boundaries of the corresponding bounded symmetric domains.

Let Aut $\left(S_{p, q}\right)$ be the Lie group of all CR automorphisms of $S_{p, q}$. By [KZ00, Theorem 8.5], every $\varphi \in$ Aut $\left(S_{p, q}\right)$ extends to a biholomorphic automorphism of the bounded symmetric domain $D_{p, q}$. Consider the standard linear inclusion

$$
z \mapsto\left(\begin{array}{c}
I_{q} \\
z
\end{array}\right), z \in S_{p, q}
$$


Then we may regard $S_{p, q}$ as a real submanifold in the Grassmanian $G r(q, p+q)$ of all $q$-planes in $\mathbb{C}^{p+q}$ and Aut $\left(S_{p, q}\right)=$ Aut $\left(D_{p, q}\right)$ becomes a subgroup of the automorphism group of $G r(q, p+q)$. In this section we will construct a frame bundle over $S_{p, q}$ associated with the CR structure of $S_{p, q}$ using Grassmannian frames of $\operatorname{Gr}(q, p+q)$.

As before, consider the partial $C R$ dimension $n=p-q$. The actual CR dimension of $S_{p, q}$ is $(p-q) q=n q$ and $q=r$ is the rank of the bounded symmetric domain $D_{p, q}$.

For column vectors $u=\left(u_{1}, \ldots, u_{p+q}\right)^{t}$ and $v=\left(v_{1}, \ldots, v_{p+q}\right)^{t}$ in $\mathbb{C}^{p+q}$, define the Hermitian inner product by

$$
\langle u, v\rangle:=-\left(u_{1} \bar{v}_{1}+\cdots+u_{q} \bar{v}_{q}\right)+\left(u_{q+1} \bar{v}_{q+1}+\cdots+u_{p+q} \bar{v}_{p+q}\right) .
$$

A Grassmannian frame adapted to $S_{p, q}$, or simply $S_{p, q^{-}}$frame is a frame $\left\{Z_{1}, \ldots, Z_{p+q}\right\}$ of $\mathbb{C}^{p+q}$ with $\operatorname{det}\left(Z_{1}, \ldots, Z_{p+q}\right)=1$ such that

$$
\left\langle Z_{\alpha}, Z_{q+n+\beta}\right\rangle=\left\langle Z_{q+n+\beta}, Z_{\alpha}\right\rangle=\delta_{\alpha \beta}, \quad\left\langle Z_{q+j}, Z_{q+k}\right\rangle=\delta_{j k}
$$

and

$$
\left\langle Z_{\Lambda}, Z_{\Gamma}\right\rangle=0 \text { otherwise, }
$$

where the capital Greek indices $\Lambda, \Gamma, \Omega$ etc. run from 1 to $p+q$. We also use the notation $Z:=\left(Z_{1}, \ldots, Z_{q}\right), \quad X=\left(X_{1}, \ldots, X_{n}\right):=\left(Z_{q+1}, \ldots, Z_{q+n}\right), \quad Y=\left(Y_{1}, \ldots, Y_{q}\right):=\left(Z_{q+n+1} \ldots, Z_{q+p}\right)$, so that (2.2) can be rewritten as

$$
\left\langle Z_{\alpha}, Y_{\beta}\right\rangle=\left\langle Y_{\beta}, Z_{\alpha}\right\rangle=\delta_{\alpha \beta}, \quad\left\langle X_{j}, X_{k}\right\rangle=\delta_{j k}
$$

i.e. the scalar product $\langle\cdot, \cdot\rangle$ in basis $\left(Z_{\alpha}, X_{j}, Y_{\beta}\right)$ is given by the matrix

$$
\left(\begin{array}{ccc}
0 & 0 & I_{q} \\
0 & I_{n} & 0 \\
I_{q} & 0 & 0
\end{array}\right)
$$

Let $\mathcal{B}_{p, q}$ be the set of all $S_{p, q}$-frames. Then $\mathcal{B}_{p, q}$ can be identified with $S U(p, q)$ by the left action. The Maurer-Cartan form $\pi=\left(\pi_{\Lambda}^{\Gamma}\right)$ on $\mathcal{B}_{p, q}$ is given by the equation

$$
d Z_{\Lambda}=\pi_{\Lambda}^{\Gamma} Z_{\Gamma}
$$

where $\pi$ satisfies the trace-free condition

$$
\sum_{\Lambda} \pi_{\Lambda}^{\Lambda}=0
$$

and the structure equation

$$
d \pi_{\Lambda}^{\Gamma}=\pi_{\Lambda}^{\Omega} \wedge \pi_{\Omega}^{\Gamma}
$$

More explicitly, using the block matrix representation with respect to the basis $(Z, X, Y)$, we can write

$$
\pi=\left(\begin{array}{ccc}
\pi_{\alpha}^{\beta} & \pi_{\alpha}^{q+j} & \pi_{\alpha}^{q+n+\beta} \\
\pi_{q+k}^{\beta} & \pi_{q+k}^{q+j} & \pi_{q+k}^{q+n+\beta} \\
\pi_{q+n+\alpha}^{\beta} & \pi_{q+n+\alpha}^{q+k} & \pi_{q+n+\alpha}^{q+n}
\end{array}\right)=:\left(\begin{array}{ccc}
\psi_{\alpha}^{\beta} & \theta_{\alpha}^{j} & \varphi_{\alpha}^{\beta} \\
\sigma_{k}^{\beta} & \omega_{k}^{j} & \theta_{k}^{\beta} \\
\xi_{\alpha}^{\beta} & \sigma_{\alpha}^{j} & \widehat{\psi}_{\alpha}^{\beta}
\end{array}\right)
$$


which satisfies the symmetry relations

$$
\left(\begin{array}{ccc}
\psi_{\alpha}^{\beta} & \theta_{\alpha}^{j} & \varphi_{\alpha}^{\beta} \\
\sigma_{k}{ }^{\beta} & \omega_{k}^{j} & \theta_{k}^{\beta} \\
\xi_{\alpha}{ }^{\beta} & \sigma_{\alpha}{ }^{j} & \widehat{\psi}_{\alpha}^{\beta}
\end{array}\right)=-\left(\begin{array}{ccc}
\widehat{\psi}_{\bar{\beta}}^{\bar{\alpha}} & \theta_{\bar{j}}^{\bar{\alpha}} & \varphi_{\bar{\beta}}^{\bar{\alpha}} \\
\sigma_{\bar{\beta}}{ }^{\bar{k}} & \omega_{\bar{j}}^{\bar{k}} & \theta_{\overline{\bar{\beta}}} \\
\xi_{\overline{\bar{\beta}}}^{\bar{\alpha}} & \sigma_{\bar{j}}^{\bar{\alpha}} & \psi_{\overline{\bar{\beta}}}^{\bar{\alpha}}
\end{array}\right)
$$

that follow directly by differentiating (2.2).

The structure equations (2.6) can be rewritten as

$$
\begin{aligned}
& d \varphi_{\alpha}{ }^{\beta}=\psi_{\alpha}^{\gamma} \wedge \varphi_{\gamma}{ }^{\beta}+\theta_{\alpha}^{l} \wedge \theta_{l}{ }^{\beta}+\varphi_{\alpha}^{\gamma} \wedge \widehat{\psi}_{\gamma}{ }^{\beta} \\
& d \theta_{\alpha}^{j}=\psi_{\alpha}^{\gamma} \wedge \theta_{\gamma}^{j}+\theta_{\alpha}^{l} \wedge \omega_{l}^{j}+\varphi_{\alpha}^{\gamma} \wedge \sigma_{\gamma}^{j} \\
& d \psi_{\alpha}{ }^{\beta}=\psi_{\alpha}{ }^{\gamma} \wedge \psi_{\gamma}{ }^{\beta}+\theta_{\alpha}{ }^{l} \wedge \sigma_{l}{ }^{\beta}+\varphi_{\alpha}^{\gamma} \wedge \xi_{\gamma}{ }^{\beta} \\
& d \omega_{k}^{j}=\sigma_{k}^{\gamma} \wedge \theta_{\gamma}^{j}+\omega_{k}^{l} \wedge \omega_{l}^{j}+\theta_{k}^{\gamma} \wedge \sigma_{\gamma}^{j} \\
& d \sigma_{k}{ }^{\beta}=\sigma_{k}^{\gamma} \wedge \psi_{\gamma}{ }^{\beta}+\omega_{k}{ }^{l} \wedge \sigma_{l}{ }^{\beta}+\theta_{k}{ }^{\gamma} \wedge \xi_{\gamma}{ }^{\beta} \\
& d \xi_{\alpha}{ }^{\beta}=\xi_{\alpha}{ }^{\gamma} \wedge \psi_{\gamma}{ }^{\beta}+\sigma_{\alpha}{ }^{l} \wedge \sigma_{l}{ }^{\beta}+\widehat{\psi}_{\alpha}{ }^{\gamma} \wedge \xi_{\gamma}{ }^{\beta} \text {, }
\end{aligned}
$$

in particular,

$$
d \varphi_{\alpha}^{\beta}=\theta_{\alpha}^{j} \wedge \theta_{j}^{\beta} \bmod \varphi,
$$

where $\varphi$ is the span of $\varphi_{\alpha}^{\beta}$ for all $\alpha, \beta$.

By abuse of notation, we also denote by $Z$ the $q$-dimensional subspace of $\mathbb{C}^{p+q}$ spanned by $Z_{1}, \ldots, Z_{q}$. Hence $Z$ represents a point in $S_{p, q}$ and vice versa, any point in $S_{p, q}$ is represented by $Z$ corresponding to an adapted frame $(Z, X, Y)$. Then $\mathcal{B}_{p, q}$ can be regarded as a bundle over $S_{p, q}$ via the projection map $(Z, X, Y) \rightarrow Z$. By another abuse of notation, we shall also use the same letters for the components of $\pi$ and their pullbacks to $S_{p, q}$ via a fixed section. Note that fixing a section means precisely choosing an adapted frame $(Z, X, Y)$ at every point $x$ of (an open subset of) $S_{p, q}$ such that $Z$ represents $x$ as a point in the Grassmanian.

The defining equations of $S_{p, q}$ can be written as

$$
S_{p, q}=\left\{[V] \in G r(q, p+q):\left.\langle\cdot, \cdot\rangle\right|_{V}=0\right\}
$$

and hence their differentiation yields

$$
\left\langle d Z_{\Lambda}, Z_{\Gamma}\right\rangle+\left\langle Z_{\Lambda}, d Z_{\Gamma}\right\rangle=0 .
$$

By substituting $d Z_{\Lambda}=\pi_{\Lambda}^{\Gamma} Z_{\Gamma}$ into $(1,0)$ component of (2.15) we obtain, in particular,

$$
\varphi_{\alpha}^{\gamma}\left\langle Y_{\gamma}, Z_{\beta}\right\rangle=\varphi_{\alpha}^{\beta}=0
$$

when restricted to the $(1,0)$ tangent space. Comparing the dimensions, we conclude that the kernel of $\left\{\varphi_{\alpha}^{\beta}, \alpha, \beta=1, \ldots, q\right\}$ forms the $\mathrm{CR}$ bundle of $S_{p, q}$, i.e.,

$$
\operatorname{ker}\left(\left.\varphi\right|_{Z}\right)=T_{Z}^{1,0} S_{p, q} \oplus T_{Z}^{0,1} S_{p, q}
$$

In other words, $\varphi=\left(\varphi_{\alpha}^{\beta}\right)$ span the space of contact forms on $S_{p, q}$. Since

$$
d Z_{\alpha}=\psi_{\alpha}{ }^{\beta} Z_{\beta}+\varphi_{\alpha}{ }^{\beta} Y_{\beta}+\theta_{\alpha}{ }^{j} X_{j}
$$


and $\varphi=\left(\varphi_{\alpha}^{\beta}\right)$ is a contact form at $Z=\left(Z_{1}, \ldots, Z_{q}\right)$, we conlcude that $\varphi_{\alpha}^{\beta}$ and $\theta_{\alpha}^{j}$ form together a basis in the space of all $(1,0)$ forms.

For a change of frame given by

$$
\left(\begin{array}{c}
\widetilde{Z} \\
\widetilde{X} \\
\widetilde{Y}
\end{array}\right):=U\left(\begin{array}{c}
Z \\
X \\
Y
\end{array}\right)
$$

$\pi$ changes via

$$
\widetilde{\pi}=d U \cdot U^{-1}+U \cdot \pi \cdot U^{-1} .
$$

There are several types of frame changes.

Definition 2.3. We call a change of frame

i) change of position if

$$
\widetilde{Z}_{\alpha}=W_{\alpha}^{\beta} Z_{\beta}, \quad \widetilde{Y}_{\alpha}=V_{\alpha}^{\beta} Y_{\beta}, \quad \widetilde{X}_{j}=X_{j},
$$

where $W=\left(W_{\alpha}{ }^{\beta}\right)$ and $V=\left(V_{\alpha}{ }^{\beta}\right)$ are $q \times q$ matrices satisfying $V^{*} W=I_{q}$;

ii) change of real vectors if

$$
\widetilde{Z}_{\alpha}=Z_{\alpha}, \quad \widetilde{X}_{j}=X_{j}, \quad \widetilde{Y}_{\alpha}=Y_{\alpha}+H_{\alpha}^{\beta} Z_{\beta},
$$

or

$$
\left(\begin{array}{c}
\widetilde{Z}_{\alpha} \\
\widetilde{X}_{j} \\
\widetilde{Y}_{\alpha}
\end{array}\right)=\left(\begin{array}{ccc}
I_{q} & 0 & 0 \\
0 & I_{n} & 0 \\
H_{\alpha}{ }^{\beta} & 0 & I_{q}
\end{array}\right)\left(\begin{array}{c}
Z_{\beta} \\
X_{k} \\
Y_{\beta}
\end{array}\right),
$$

where $H=\left(H_{\alpha}^{\beta}\right)$ is a hermitian matrix;

iii) dilation if

$$
\widetilde{Z}_{\alpha}=\lambda_{\alpha}^{-1} Z_{\alpha}, \quad \tilde{Y}_{\alpha}=\lambda_{\alpha} Y_{\alpha}, \quad \tilde{X}_{j}=X_{j}
$$

where $\lambda_{\alpha}>0$

iv) rotation if

$$
\widetilde{Z}_{\alpha}=Z_{\alpha}, \quad \widetilde{Y}_{\alpha}=Y_{\alpha}, \quad \tilde{X}_{j}=U_{j}{ }^{k} X_{k},
$$

where $\left(U_{j}{ }^{k}\right)$ is a unitary matrix.

Consider a change of position as in Definition 2.3. Then $\varphi$ and $\theta$ change to

$$
\widetilde{\varphi}_{\alpha}^{\beta}=W_{\alpha}^{\gamma} \varphi_{\gamma}^{\delta} W_{\delta}^{*}, \quad W_{\delta}^{*}=\overline{W_{\beta}^{\delta}}, \quad \tilde{\theta}_{\alpha}{ }^{j}=W_{\alpha}^{\beta} \theta_{\beta}^{j} .
$$

We shall also make use of the change of frame given by

$$
\widetilde{Z}_{\alpha}=Z_{\alpha}, \quad \tilde{X}_{j}=X_{j}+C_{j}{ }^{\beta} Z_{\beta}, \quad \tilde{Y}_{\alpha}=Y_{\alpha}+A_{\alpha}{ }^{\beta} Z_{\beta}+B_{\alpha}{ }^{j} X_{j},
$$

or

$$
\left(\begin{array}{c}
\widetilde{Z}_{\alpha} \\
\widetilde{X}_{j} \\
\widetilde{Y}_{\alpha}
\end{array}\right)=\left(\begin{array}{ccc}
I_{q} & 0 & 0 \\
C_{j}{ }^{\beta} & I_{n} & 0 \\
A_{\alpha}^{\beta} & B_{\alpha}{ }^{j} & I_{q}
\end{array}\right)\left(\begin{array}{c}
Z_{\beta} \\
X_{k} \\
Y_{\beta}
\end{array}\right),
$$


such that

$$
C_{j}^{\alpha}+B_{j}^{\alpha}=0
$$

and

$$
\left(A_{\alpha}^{\beta}+\overline{A_{\beta}^{\alpha}}\right)+B_{\alpha}{ }^{j} B_{j}{ }^{\beta}=0,
$$

where

$$
B_{j}^{\alpha}:=\overline{B_{\alpha}^{j}} .
$$

Then the new frame $(\widetilde{Z}, \widetilde{Y}, \widetilde{X})$ is an $S_{p, q}$-frame. In fact,

$$
\begin{aligned}
0=\left\langle\tilde{Y}_{\alpha}, \tilde{Y}_{\beta}\right\rangle & =\left\langle Y_{\alpha}+A_{\alpha}{ }^{\delta} Z_{\delta}+B_{\alpha}{ }^{j} X_{j}, Y_{\beta}+A_{\beta}^{\gamma} Z_{\gamma}+B_{\beta}{ }^{k} X_{k}\right\rangle \\
& =A_{\alpha}{ }^{\beta}\left\langle Z_{\beta}, Y_{\beta}\right\rangle+\overline{A_{\beta}^{\alpha}}\left\langle Y_{\alpha}, Z_{\alpha}\right\rangle+\sum_{j} B_{\alpha}{ }^{j} \overline{B_{\beta}^{j}}\left\langle X_{j}, X_{j}\right\rangle=\left(A_{\alpha}{ }^{\beta}+\overline{A_{\beta}{ }^{\alpha}}\right)+\sum_{j} B_{\alpha}{ }^{j} \overline{B_{\beta}^{j}},
\end{aligned}
$$

and

$$
0=\left\langle\widetilde{X}_{j}, \widetilde{Y}_{\alpha}\right\rangle=\left\langle X_{j}+C_{j}{ }^{\beta} Z_{\beta}, Y_{\alpha}+A_{\alpha}{ }^{\delta} Z_{\delta}+B_{\alpha}{ }^{k} X_{k}\right\rangle=C_{j}{ }^{\alpha}\left\langle Z_{\alpha}, Y_{\alpha}\right\rangle+\overline{B_{\alpha}{ }^{j}}\left\langle X_{j}, X_{j}\right\rangle=C_{j}{ }^{\alpha}+\overline{B_{\alpha}{ }^{j}},
$$

whereas the other scalar products are obviously zero. Furthermore, we claim that the related 1-forms $\widetilde{\varphi}_{\alpha}^{\beta}$ remain the same, while $\widetilde{\theta}_{\alpha}^{j}$ change to

$$
\tilde{\theta}_{\alpha}^{j}=\theta_{\alpha}^{j}-\varphi_{\alpha}^{\beta} B_{\beta}^{j}
$$

Indeed, differentiation yields

$$
\begin{gathered}
d \widetilde{Z}_{\alpha}=\widetilde{\psi}_{\alpha}{ }^{\beta} \widetilde{Z}_{\beta}+\widetilde{\theta}_{\alpha}^{j} \widetilde{X}_{j}+\widetilde{\varphi}_{\alpha}{ }^{\beta} \widetilde{Y}_{\beta}=\widetilde{\psi}_{\alpha}{ }^{\beta} Z_{\beta}+\tilde{\theta}_{\alpha}{ }^{j}\left(X_{j}+C_{j}{ }^{\beta} Z_{\beta}\right)+\widetilde{\varphi}_{\alpha}{ }^{\beta}\left(Y_{\beta}+A_{\beta}{ }^{\gamma} Z_{\gamma}+B_{\beta}{ }^{j} X_{j}\right) \\
=d Z_{\alpha}=\psi_{\alpha}{ }^{\beta} Z_{\beta}+\theta_{\alpha}^{j} X_{j}+\varphi_{\alpha}{ }^{\beta} Y_{\beta}
\end{gathered}
$$

and the claim follows from identifying the coefficients.

\section{CARTAN's LEMMA}

We shall routinely use the Cartan's Lemma for complex-valued forms:

Lemma 3.1 (Cartan's Lemma). Let $\theta_{1}, \ldots, \theta_{r}$ be complex-linearly independent complex-valued 1forms on a real manifold $M$ and $\varphi_{1}, \ldots, \varphi_{r}$ be further complex-valued 1-forms on $M$ satisfying

$$
\theta_{1} \wedge \varphi_{1}+\ldots+\theta_{r} \wedge \varphi_{r}=0 \text {. }
$$

Then

$$
\varphi_{j}=0 \bmod \left\{\theta_{1}, \ldots, \theta_{r}\right\}
$$

for each $j=1, \ldots, r$. 
Proof. Complete $\theta_{1}, \ldots, \theta_{r}$ to a basis $\theta_{1}, \ldots, \theta_{s}$ in the space of all complex-valued 1-forms on $M$. Then we can write

$$
\varphi_{j}=c_{j}^{k} \theta_{k}
$$

for suitable coefficients $c_{j}^{k}$. Then substituting into (3.1), using the fact that the set of $\theta_{i} \wedge \theta_{j}$ with $i<j$ is a basis in the space of all 2-forms, and identifying coefficients of $\theta_{j} \wedge \theta_{k}$ for $j \leq r, k>r$, we conlcude

$$
c_{j}^{k}=0, \quad j \leq r<k,
$$

and the claim follows.

\section{Determination of $\Phi_{a}^{b}$ And $\Theta_{a}{ }^{J}$ Modulo $\varphi$ Using the LeVi Form identities.}

Let $p>q, p^{\prime}>q^{\prime}$ be positive integers and let $f$ be a local CR embedding from $S_{p, q}$ into $S_{p^{\prime}, q^{\prime}}$ Denote by $\mathcal{B}_{p, q}$ and $\mathcal{B}_{p^{\prime}, q^{\prime}}$ the Grassmannian frame bundles adapted to $S_{p, q}$ and $S_{p^{\prime}, q^{\prime}}$ respectively. We set $n:=p-q, n^{\prime}:=p^{\prime}-q^{\prime}$ and follow the index convention at the beginning of $\S 2$.

We shall consider the connection forms $\varphi_{\alpha}^{\beta}, \theta_{\alpha}{ }^{j}, \psi_{\alpha}{ }^{\beta}, \omega_{j}{ }^{k}, \sigma_{j}{ }^{\beta}, \xi_{\alpha}{ }^{\beta}$ on $\mathcal{B}_{p, q}$ pulled back to $S_{p, q}$ and denote by capital letters $\Phi_{a}^{b}, \Theta_{a}{ }^{J}, \Psi_{a}^{b}, \Omega_{J}^{K}, \Sigma_{K}^{b}, \Xi_{a}^{b}$ their corresponding counterparts on $\mathcal{B}_{p^{\prime}, q^{\prime}}$ pulled back to $S_{p^{\prime}, q^{\prime}}$. Furthermore, we shall adopt the convention that any form is assumed to be zero whenever its indices are out of the range where the form is defined, e.g. $\theta_{a}{ }^{J}=0$ if either $a>q$ or $J>n$, or $\varphi_{a}^{b}=0$ if either $a>q$ or $b>q$.

Since $\varphi=\left(\varphi_{\alpha}^{\beta}\right)$ and $\Phi=\left(\Phi_{a}^{b}\right)$ are contact forms on $S_{p, q}$ and $S_{p^{\prime}, q^{\prime}}$, respectively, the pull back of $\Phi$ via $f$ is a linear combination of $\varphi=\left(\varphi_{\alpha}^{\beta}\right)$.

We shall abuse the notation by writing $\Sigma$ instead of $f^{*} \Sigma$ for any form $\Sigma$ on $S_{p^{\prime}, q^{\prime}}$. Thus all our forms will be understood on $S_{p, q}$ and any form on $S_{p^{\prime}, q^{\prime}}$ will be assumed pulled back to $S_{p, q}$ via the given $\mathrm{CR}$ map $f$ without explicit mentioning.

In this section our analysis will be based on using the structure equation for $\varphi$ modulo the ideal generated by the contact forms $\varphi_{\alpha}^{\beta}$, i.e. on the equations

$$
d \varphi_{a}^{b}=\theta_{a}^{j} \wedge \theta_{j}^{b} \bmod \varphi, \quad d \Phi_{a}^{b}=\Theta_{\alpha}^{J} \wedge \Theta_{J}^{b} \bmod \varphi .
$$

By writing identities modulo $\varphi$ we shall always mean that the difference between the left- and righthand sides is contained in the ideal generated by the components $\varphi_{\alpha}^{\beta}$ in the exterior algebra. In the second identity we have also used the fact mentioned above that (the pullback of) any $\Phi_{a}^{b}$ is a linear combination of $\varphi_{\alpha}^{\beta}$. Note that due to our convention, both sides of the first equation are zero if either $a>q$ or $b>q$ and for the same reason the summation is only performed over $j \in\{1, \ldots, n\}$.

4.1. Determination of $\Phi_{1}{ }^{1}$. Consider the diagonal terms $\Phi_{a}^{a}, a=1, \ldots, q^{\prime}$. Suppose that (the pullbacks of) $\Phi_{a}{ }^{a}$ vanish identically for all $a$. Then (4.1) yields

$$
0=d \Phi_{a}^{a}=-\sum_{J} \Theta_{a}^{J} \wedge \overline{\Theta_{a}^{J}} \bmod \varphi .
$$

Since each $\Theta_{a}^{J}$ is a $(1,0)$ form and each wedge product is non-negative on $(T, \bar{T})$ where $T$ is any $(1,0)$ vector, it follows that

$$
\Theta_{a}{ }^{J}=0 \bmod \varphi,
$$

which contradicts the assumption that $f$ is an embedding. 
Hence there exists at least one diagonal term of $\Phi$ whose pullback does not vanish identically. Choose such a diagonal term of $\Phi$, say $\Phi_{1}{ }^{1}$. Then on an open set, $\Phi_{1}{ }^{1} \neq 0$. Since the pullback of $\Phi_{1}^{1}$ to $S_{p, q}$ is a contact form, we can write

$$
\Phi_{1}^{1}=c_{\alpha}^{\beta} \varphi_{\beta}^{\alpha}
$$

for some smooth functions $c_{\alpha}{ }^{\beta}$. Since $\left(\varphi_{\alpha}{ }^{\beta}\right)$ and $\left(\Phi_{a}{ }^{b}\right)$ are antihermitian, the matrix $\left(c_{\alpha}{ }^{\beta}\right)$ is hermitian. Then there exists a change of frame on $S_{p, q}$ (change of position in Definition 2.3) given by

$$
\widetilde{Z}_{\alpha}=U_{\alpha}{ }^{\beta} Z_{\beta}, \tilde{Y}_{\alpha}=U_{\alpha}{ }^{\beta} Y_{\beta}, \widetilde{X}_{j}=X_{j},
$$

for some unitary matrix $U$ such that $c_{\alpha}^{\beta}$ is diagonalized and hence the new contact forms $\varphi_{\alpha}^{\beta}, \alpha, \beta=$ $1, \ldots, q$, satisfy

$$
\Phi_{1}^{1}=\sum_{\alpha=1}^{r} c_{\alpha} \varphi_{\alpha}^{\alpha}, \quad 1 \leq r \leq q,
$$

where $c_{\alpha}, \alpha=1, \ldots, r$, are nonzero real valued smooth functions. Then (4.1) yields

$$
\sum_{J} \Theta_{1}^{J} \wedge \overline{\Theta_{1}^{J}}=\sum_{\alpha, j} c_{\alpha} \theta_{\alpha}^{j} \wedge \overline{\theta_{\alpha}^{j}} \bmod \varphi
$$

which implies $c_{\alpha}>0$ in view of the non-negativity mentioned above since the forms $\theta_{\alpha}^{\gamma} \wedge \overline{\theta_{\alpha}^{\gamma}}$ are linearly independent. Hence after dilation of $\Phi_{1}{ }^{1}$, we may assume that

$$
c_{1}=1 \text {. }
$$

Lemma 4.1. Assuming $n^{\prime}<2 n$, we have $r=1$ and

$$
\begin{aligned}
\Phi_{1}{ }^{1} & =\varphi_{1}{ }^{1}, \\
\Theta_{1}{ }^{J} & =\theta_{1}{ }^{J} \quad \bmod \varphi .
\end{aligned}
$$

Proof. Let

$$
\Theta_{1}^{J}=h_{j}^{J, \alpha} \theta_{\alpha}^{j} \bmod \varphi .
$$

Then (4.2) implies

$$
\sum_{J} h_{j}^{J, \alpha} \overline{h_{k}^{J, \beta}}=c_{\alpha} \delta_{\alpha \beta} \cdot \delta_{j k}
$$

where $c_{\alpha}:=0$ for $\alpha>r$. Thus the vectors $h_{j}^{\alpha}:=\left(h_{j}^{1, \alpha}, \ldots, h_{j}^{n^{\prime}, \alpha}\right)$ are pairwise orthogonal and have length $c_{\alpha}$ independent of $j$. Therefore after a suitable rotation (see Definition 2.3)

$$
\widetilde{\Theta}_{a}{ }^{J}=\Theta_{a}{ }^{K} U_{K}^{J}
$$

where $\left(U_{K}^{J}\right)$ is unitary, we may assume that $h^{1}{ }_{j}$, whose length is $c_{1}=1$, are precisely the first $n$ standard unit vectors in $\mathbb{C}^{n^{\prime}}$, i.e.

$$
h_{j}^{J, 1}=\delta_{J j}
$$


Then for every fixed $\alpha \neq 1$, we have $n$ orthogonal vectors $h_{j}^{\alpha}$ in the span of the last $n^{\prime}-n$ standard unit vectors. Since $n^{\prime}-n<n$ by our assumption, the latter is only possible when $h^{\alpha}{ }_{j}=0$ for all $\alpha \neq 1$. Thus we obtain

$$
h_{j}^{J, \alpha}=\delta_{J j} \delta_{1 \alpha} .
$$

Then (4.5) implies (4.4) and hence (4.2) implies $r=1$ and therefore (4.1) implies (4.3).

4.2. Determination of $\Phi_{2}{ }^{2}$ and $\Phi_{2}{ }^{1}$. Consider the ideal $\theta_{\alpha}$ generated by $\theta_{\alpha}{ }^{j}$ for $1 \leq j \leq n$. Let

$$
\Phi_{a}{ }^{1}=\lambda_{a} \varphi_{1}{ }^{1} \bmod \left\{\varphi_{\alpha}^{\beta}, \alpha \geq 2 \text { or } \beta \geq 2\right\}, \quad a \geq 2,
$$

for some smooth functions $\lambda_{a}, a=2, \ldots, q^{\prime}$. Then (4.1) together with Lemma 4.1 imply

$$
\Theta_{a}^{j} \wedge \theta_{j}{ }^{1}=\lambda_{a} \theta_{1}{ }^{j} \wedge \theta_{j}{ }^{1} \bmod \left\{\theta_{\alpha}, \overline{\theta_{\alpha}}, \alpha \geq 2\right\}, \varphi, \quad a \geq 2 .
$$

Then there exists a change of position that leaves $\Theta_{1}{ }^{J}$ invariant and replaces $\Theta_{a}{ }^{J}$ with $\Theta_{a}{ }^{J}-\lambda_{a} \Theta_{1}{ }^{J}$, $a \geq 2$, (see the discussion after Definition 2.3). The same change of position leaves $\Phi_{1}{ }^{1}$ invariant and transforms $\Phi_{a}{ }^{1}$ into $\Phi_{a}{ }^{1}-\lambda_{a} \Phi_{1}{ }^{1}$ for $a \geq 2$. After performing such change of position, (4.8) becomes

$$
\Phi_{a}{ }^{1}=0 \bmod \left\{\varphi_{\alpha}{ }^{\beta}: \alpha \geq 2 \text { or } \beta \geq 2\right\}, \quad a \geq 2,
$$

and (4.9) becomes

$$
\Theta_{a}{ }^{j} \wedge \theta_{j}{ }^{1}=0 \quad \bmod \left\{\theta_{\alpha}, \overline{\theta_{\alpha}}: \alpha \geq 2\right\}, \varphi, \quad a \geq 2
$$

Since $\Theta_{a}{ }^{j}$ are $(1,0)$ but $\theta_{j}{ }^{1}$ are $(0,1)$ and linearly independent, it follows that

$$
\Theta_{a}{ }^{j}=0 \bmod \left\{\theta_{\alpha}: \alpha \geq 2\right\}, \varphi, \quad a \geq 2 .
$$

Next for each $a \geq 2$, let

$$
\Phi_{a}^{a}=\lambda_{a, \beta} \varphi_{1}^{\beta} \bmod \left\{\varphi_{\alpha}^{\gamma}: \alpha \geq 2\right\}
$$

for some functions $\lambda_{a, \beta}$. Suppose there exists $a$ and $\beta$ such that $\lambda_{a, \beta} \neq 0$. We may assume $a=2$. Using the identity

together with (4.11) we obtain

$$
d \Phi_{2}^{2}=\Theta_{2}^{J} \wedge \Theta_{J}^{2} \bmod \Phi
$$

$$
\sum_{J=n+1}^{n^{\prime}} \Theta_{2}^{J} \wedge \Theta_{J}^{2}=\lambda_{2, \beta} \theta_{1}^{j} \wedge \theta_{j}{ }^{\beta} \bmod \left\{\theta_{\alpha}: \alpha \geq 2\right\}, \varphi,
$$

where $\lambda_{2, \beta} \neq 0$. On the left-hand side we have a linear combination of $n^{\prime}-n(1,0)$ forms, whereas on the right-hand side we have a linear combination of at least $n$ linear independent $(1,0)$ forms with nonzero coefficients. Since $n^{\prime}-n<n$, this is impossible. Hence we have $\lambda_{a, \beta}=0$ for all $a, \beta$ and therefore (4.12) implies

$$
\Phi_{a}^{a}=0 \bmod \left\{\varphi_{\alpha}^{\beta}: \alpha \geq 2\right\}, \quad a \geq 2 .
$$

Since $\Phi_{a}^{b}$ and $\varphi_{\alpha}^{\beta}$ are antihermitian, we also have

$$
\Phi_{a}^{a}=0 \bmod \left\{\varphi_{\alpha}^{\beta}: \beta \geq 2\right\}, \quad a \geq 2,
$$

and hence

$$
\Phi_{a}^{a}=0 \bmod \left\{\varphi_{\alpha}^{\beta}: \alpha, \beta \geq 2\right\}, \quad a \geq 2
$$


Now (4.1) implies

$$
\sum_{J=n+1}^{n^{\prime}} \Theta_{a}^{J} \wedge \overline{\Theta_{a}^{J}}=0 \bmod \left\{\theta_{\alpha}: \alpha \geq 2\right\}, \varphi, \quad a \geq 2
$$

which implies

$$
\Theta_{a}{ }^{J}=0 \bmod \left\{\theta_{\alpha}: \alpha \geq 2\right\}, \varphi, \quad a \geq 2, J>n .
$$

Together with (4.11) this yields

$$
\Theta_{a}{ }^{J}=0 \bmod \left\{\theta_{\alpha}: \alpha \geq 2\right\}, \varphi, \quad a \geq 2 .
$$

Now we redo our procedure for $\Phi_{a}^{b}$. We can write

$$
\Phi_{a}^{b}=\lambda_{a \beta}^{b}{ }^{\alpha} \varphi_{\alpha}^{\beta}
$$

for which (4.1) yields

$$
\Theta_{a}^{J} \wedge \Theta_{J}^{b}=\lambda_{a \beta}^{b}{ }^{\alpha} \theta_{\alpha}^{j} \wedge \theta_{j}^{\beta} \bmod \varphi .
$$

Then substituting (4.17) we obtain

$$
\lambda_{a \beta}^{b}{ }^{\alpha} \theta_{\alpha}{ }^{j} \wedge \theta_{j}{ }^{\beta}=0 \quad \bmod \left\{\theta_{\gamma}{ }^{k} \wedge \theta_{l}^{\delta}: \gamma, \delta \geq 2\right\}, \varphi, \quad a, b \geq 2,
$$

which implies

$$
\lambda_{a 1}^{b{ }^{\alpha}}=\lambda_{a \beta}^{b 1}=0, \quad a, b \geq 2 .
$$

Hence (4.18) yields

$$
\Phi_{a}{ }^{b}=0 \bmod \left\{\varphi_{\alpha}^{\beta}: \alpha, \beta \geq 2\right\}, \quad a, b \geq 2 .
$$

Summarizing we obtain the following:

$$
\begin{aligned}
\Phi_{a}{ }^{1}=0 & \bmod \left\{\varphi_{\alpha}{ }^{\beta}: \alpha \geq 2 \text { or } \beta \geq 2\right\}, \quad a \geq 2, \\
\Phi_{a}{ }^{b}=0 & \bmod \left\{\varphi_{\alpha}{ }^{\beta}: \alpha, \beta \geq 2\right\}, \quad a, b \geq 2, \\
\Theta_{a}{ }^{J}=0 & \bmod \left\{\theta_{\alpha}: \alpha \geq 2\right\}, \varphi, \quad a \geq 2 .
\end{aligned}
$$

Now repeat the argument from the beginning of this section and assume first that $\Phi_{a}^{a}=0$ for all $a \geq 2$. We obtain

$$
\Theta_{a}^{J}=0 \bmod \varphi, \quad a \geq 2,
$$

and hence $d f$ vanishes on the kernel of all $\theta_{1}^{j}$ and $\varphi_{\alpha}^{\beta}$. Since $f$ is an embedding, it follows that the latter kernel equals the full complex tangent space, i.e. $q=1$. In this case (4.16) implies

$$
\Theta_{a}^{J}=0 \bmod \varphi, \quad a>1=q .
$$

Then writing (4.18) and proceeding as before we obtain $\lambda_{a}{ }^{b}{ }^{\alpha}=0$ and hence

$$
\Phi_{a}^{b}=0, \quad q=1 .
$$

(Note that we have assumed $q \geq 2$ excluding this case. However, we shall repeat this procedure when a similar case will occur.) 
In the remaining case $q>1$, our assumption above cannot hold, i.e. $\Phi_{a}^{a} \neq 0$ for some $a$, say $a=2$. Then (4.14) implies that, after a change of position as before, we may assume that

$$
\Phi_{2}^{2}=\sum_{\alpha \geq 2} c_{\alpha} \varphi_{\alpha}^{\alpha}
$$

for some $c_{\alpha} \geq 0$ not all zero. Then (4.1) yields

$$
\Theta_{2}^{J} \wedge \Theta_{J}^{2}=\sum_{\alpha \geq 2} c_{\alpha} \theta_{\alpha}^{j} \wedge \theta_{j}^{\alpha} \bmod \varphi
$$

Since the proof of Lemma 4.1 can be repeated for $\Phi_{2}{ }^{2}$ instead of $\Phi_{1}{ }^{1}$, we conclude that the rank of the left-hand side of (4.26) restricted to the complex tangent space is $n$. Therefore, in the right-hand side, only one $c_{\alpha}$, say $c_{2}$ can be different from zero. After a dilation (see Definition 2.3), we may assume

$$
\Phi_{2}^{2}=\varphi_{2}^{2}
$$

and hence

$$
\sum_{J} \Theta_{2}^{J} \wedge \overline{\Theta_{2}{ }^{J}}=\sum_{j} \theta_{2}^{j} \wedge \overline{\theta_{2}^{j}} \bmod \varphi .
$$

We claim that each $\Theta_{2}{ }^{J}$ is a linear combination of only $\theta_{2}^{j}$ modulo $\varphi$. Indeed, if $\Theta_{2}{ }^{J}$ were a combination of $\theta_{\alpha}^{j}$ modulo $\varphi$, where some of them enters with a nonzero coefficient $\lambda_{\alpha}$ with $\alpha \neq 2$, we would have $\theta_{\alpha}{ }^{j} \wedge \overline{\theta_{\alpha}^{j}}$ entering with positive coefficient $\geq \lambda_{\alpha} \overline{\lambda_{\alpha}}$ in the right-hand side of (4.27), which is impossible, proving our claim. As in the proof of Lemma 4.1 we now write

$$
\Theta_{2}^{J}=h^{J} \theta_{2}^{j} \bmod \varphi \text {. }
$$

Since

$$
\Phi_{2}^{1}=\lambda_{\beta}^{\alpha} \varphi_{\alpha}^{\beta}
$$

for suitable $\lambda_{\alpha}{ }^{\beta}$, we obtain

$$
\Theta_{2}{ }^{J} \wedge \Theta_{J}^{1}=\lambda_{\beta}^{\alpha} \theta_{\alpha}{ }^{j} \wedge \theta_{j}{ }^{\beta} \bmod \varphi,
$$

which in view of (4.28) and Lemma 4.1, yields

$$
h^{k}{ }_{j} \theta_{2}^{j} \wedge \theta_{k}{ }^{1}=\lambda_{\beta}^{\alpha} \theta_{\alpha}^{j} \wedge \theta_{j}^{\beta} \bmod \varphi .
$$

Since the right-hand side contains no terms $\theta_{\alpha}{ }^{j} \wedge \theta_{k}{ }^{\beta}$ with $j \neq k$, it follows that $h^{k}{ }_{j}=0$ for $j \neq k$ and hence $h^{j}{ }_{j}=\lambda_{2}{ }^{1}=: \lambda$ for all $j$ and $\lambda_{\beta}{ }^{\alpha}=0$ for $(\alpha, \beta) \neq(1,2)$. Then (4.28) implies

$$
\Theta_{2}^{j}=\lambda \theta_{2}^{j} \bmod \varphi \text {. }
$$

Finally, substituting (4.32) into (4.27) and identifying coefficients we obtain

$$
\lambda \bar{\lambda} \delta_{i j}+\sum_{J>n} h_{i}^{J} \bar{h}_{j}^{J}=\delta_{i j}
$$

In particular, it follows that the vectors $h_{i}:=\left(h_{i}^{n+1}, \ldots, h_{i}^{n^{\prime}}\right)$ are orthogonal and of the same length. But since we have assumed $n^{\prime}-n<n$, we must have $h_{i}=0$ and therefore $|\lambda|=1$. Now we perform 
a change of position as in Definition 2.3 with $W_{\alpha}{ }^{\beta}:=c_{\alpha} \delta_{\alpha \beta}$ with $c_{\alpha}=1$ for $\alpha \neq 2$ and $c_{2}=\lambda$. Then we arrive at the following relations:

$$
\begin{gathered}
\Phi_{a}{ }^{a}=\varphi_{a}{ }^{a}, \quad a=1,2, \\
\Theta_{a}{ }^{J}=\theta_{a}{ }^{J} \quad \bmod \varphi, \quad a=1,2 .
\end{gathered}
$$

Since after the last change of position, we have $\lambda=1$ in (4.32), we obtain from (4.31) that $\lambda_{\beta}{ }^{\alpha}=0$ unless $\alpha=2$ and $\beta=1$, in which case $\lambda_{2}{ }^{1}=1$. Then substituting into (4.29) yields

$$
\Phi_{2}^{1}=\varphi_{2}^{1} \text {. }
$$

4.3. Determination of $\Phi_{a}^{b}$. Now we repeat again the arguments after the proof of Lemma 4.1, where we replace 1 by 2 and 2 by 3 , to arrive at the identities:

$$
\begin{aligned}
& \Phi_{a}{ }^{2}=0 \quad \bmod \left\{\varphi_{\alpha}{ }^{\beta}: \alpha \geq 3 \text { or } \beta \geq 3\right\}, \quad a \geq 3, \\
& \Phi_{a}{ }^{b}=0 \quad \bmod \left\{\varphi_{\alpha}{ }^{\beta}: \alpha, \beta \geq 3\right\}, \quad a, b \geq 3, \\
& \Theta_{a}{ }^{J}=0 \bmod \left\{\theta_{\alpha}: \alpha \geq 3\right\}, \varphi, \quad a \geq 3 .
\end{aligned}
$$

Then continuing following the arguments after (4.24) with the same replacements, we obtain

$$
\begin{gathered}
\Phi_{3}{ }^{\alpha}=\varphi_{3}{ }^{\alpha}, \quad \alpha=1,2,3, \\
\Theta_{3}{ }^{J}=\theta_{3}{ }^{J} \quad \bmod \varphi .
\end{gathered}
$$

Finally, arguing by induction on $b=4, \ldots, q^{\prime}$, and proceeding by repeating the same arguments with 1 replaced by $b$ and 2 by $b+1$, we obtain the following lemma.

Lemma 4.2. For any local $C R$ embedding $f$ from $S_{p, q}$ into $S_{p^{\prime}, q^{\prime}}$, there is a choice of sections of the bundles $\mathcal{B}_{p, q} \rightarrow S_{p, q}$ and $\mathcal{B}_{p^{\prime}, q^{\prime}} \rightarrow S_{p^{\prime}, q^{\prime}}$ such that the pulled back forms satisfy

$$
\begin{aligned}
\Phi_{a}{ }^{b}-\varphi_{a}{ }^{b} & =0, \\
\Theta_{a}{ }^{J}-\theta_{a}{ }^{J} & =0 \bmod \varphi .
\end{aligned}
$$

Remark 4.3. A change of section of $\mathcal{B}_{p, q} \rightarrow S_{p, q}$ (corresponding to a change of frame on $S_{p, q}$ ) has been used in course of the proof. However, once Lemma 4.2 has been established, one can change the frame on $S_{p, q}$ back to the original one together with the corresponding change of the frame on $S_{p^{\prime}, q^{\prime}}$ involving only the subframe $\left(Z_{a}, X_{J}, Y_{b}\right)$ with $a, b \leq q, J \leq n$, such that the conclusion of the lemma remains valid.

\section{Determination of $\Theta_{a}^{J}$.}

In our next analysis we shall use the full structure equations for $\varphi$ and $\Phi$ which in view of Lemma 4.2 take the form

$$
\begin{aligned}
& d \varphi_{a}^{b}=\psi_{a}^{\gamma} \wedge \varphi_{\gamma}^{b}+\theta_{a}^{j} \wedge \theta_{j}^{b}+\varphi_{a}^{\gamma} \wedge \widehat{\psi}_{\gamma}^{b}, \\
& d \varphi_{a}^{b}=\Psi_{a}^{\gamma} \wedge \varphi_{\gamma}^{b}+\Theta_{a}{ }^{J} \wedge \Theta_{J}^{b}+\varphi_{a}^{\gamma} \wedge \widehat{\Psi}_{\gamma}^{b},
\end{aligned}
$$

and their difference

$$
\left(\Psi_{a}^{\gamma}-\psi_{a}^{\gamma}\right) \wedge \varphi_{\gamma}^{b}+\Theta_{a}^{J} \wedge \Theta_{J}^{b}-\theta_{a}^{j} \wedge \theta_{j}^{b}+\varphi_{a}^{\gamma} \wedge\left(\widehat{\Psi}_{\gamma}^{b}-\widehat{\psi}_{\gamma}^{b}\right)=0
$$


as well as the structure equations for $\theta$ and $\Theta$ :

$$
\begin{aligned}
d \theta_{a}{ }^{J} & =\psi_{a}{ }^{\beta} \wedge \theta_{\beta}{ }^{J}+\theta_{a}{ }^{k} \wedge \omega_{k}{ }^{J}+\varphi_{a}{ }^{\beta} \wedge \sigma_{\beta}{ }^{J}, \\
d \Theta_{a}{ }^{J} & =\Psi_{a}{ }^{b} \wedge \Theta_{b}{ }^{J}+\Theta_{a}{ }^{K} \wedge \Omega_{K}{ }^{J}+\varphi_{a}{ }^{\beta} \wedge \Sigma_{\beta}{ }^{J} .
\end{aligned}
$$

Our next goal is to determine $\Theta_{a}^{J}$. It will be determined together with components $\Psi_{a}^{b}$ and $\Omega_{K}^{J}$ modulo $\varphi$. In view of Lemma 4.2 we can write

$$
\Theta_{a}^{J}-\theta_{a}^{J}=\eta_{a}^{J}{ }_{\beta}^{\gamma} \varphi_{\gamma}^{\beta}
$$

for some $\eta_{a}^{J}{ }_{\beta}^{\gamma}$, and using the symmetry relations (2.8),

$$
\Theta_{J}^{a}-\theta_{J}^{a}=\eta_{J}^{a}{ }_{\gamma}^{\beta} \varphi_{\beta}^{\gamma}
$$

where

$$
\eta_{J \gamma}^{a \beta}:=\overline{\eta_{a \beta}^{J \gamma}}
$$

We will show that after a frame change, we may assume that

$$
\eta_{a}^{J}{ }_{\beta}^{\gamma}=0 .
$$

Using (5.6) we compute

$$
\Theta_{a}^{J} \wedge \Theta_{J}^{b}-\theta_{a}^{j} \wedge \theta_{j}^{b}=\eta_{a}^{j}{ }_{\beta}^{\gamma} \varphi_{\gamma}^{\beta} \wedge \theta_{j}^{b}-\theta_{a}^{j} \wedge \eta_{j}{ }^{b}{ }_{\gamma}^{\beta} \varphi_{\beta}^{\gamma} \quad \bmod \varphi \wedge \varphi
$$

and (5.3) becomes

$$
\left(\Psi_{a}^{\gamma}-\psi_{a}^{\gamma}\right) \wedge \varphi_{\gamma}^{b}+\varphi_{a}^{\gamma} \wedge\left(\widehat{\Psi}_{\gamma}^{b}-\widehat{\psi}_{\gamma}^{b}\right)+\eta_{a}^{j}{ }_{\beta}^{\gamma} \varphi_{\gamma}^{\beta} \wedge \theta_{j}^{b}-\theta_{a}^{j} \wedge \eta_{j}^{b}{ }_{\gamma}^{\beta} \varphi_{\beta}^{\gamma}=0, \quad \bmod \varphi \wedge \varphi
$$

where $\varphi \wedge \varphi$ stands for the space generated by all possible wedge products $\varphi_{\beta}^{\gamma} \wedge \varphi_{\delta}^{\tau}$. Together with (5.10) we shall consider the structure equations obtained by differentiating (5.6) and using (4.1), (5.4) and (5.5):

$$
\eta_{a}{ }^{J}{ }^{\gamma} \theta_{\gamma}^{k} \wedge \theta_{k}^{\beta}=\left(\Psi_{a}^{\beta}-\psi_{a}^{\beta}\right) \wedge \theta_{\beta}^{J}+\theta_{a}^{k} \wedge\left(\Omega_{k}^{J}-\omega_{k}^{J}\right) \bmod \varphi
$$

5.1. Determination of $\Theta_{a}{ }^{J}$ for $a>q, J>n$. In case $a>q$ and $J>n$ the right-hand side of (5.11) is zero. Since the forms $\theta_{\gamma}{ }^{k}$ and $\theta_{k}{ }^{\beta}$ are $(1,0)$ and $(0,1)$ respectively and are linearly independent, we conclude

$$
\eta_{a \beta}^{J \gamma}=0, \quad a>q, J>n
$$

and hence (5.6) yields

$$
\Theta_{a}^{J}=0, \quad a>q, J>n
$$


5.2. Determination of $\Theta_{a}^{j}$ for $a>q$. For $a>q, b=\beta \leq q$, (5.10) takes the form

$$
0=\Psi_{a}^{\alpha} \wedge \varphi_{\alpha}^{\beta}+\eta_{a}^{j} \delta^{\alpha} \varphi_{\alpha}^{\delta} \wedge \theta_{j}^{\beta} \bmod \varphi \wedge \varphi, \quad a>q
$$

Since the forms $\Re\left(\varphi_{\beta}^{\alpha}\right), \alpha<\beta$, and $\Im\left(\varphi_{\beta}^{\alpha}\right), \alpha \leq \beta$, are linearly independent over $\mathbb{R}$, it follows that $\varphi_{\alpha}^{\beta}$ are linearly independent over $\mathbb{C}$. Then we can collect the coefficients in front of these forms and apply complex Cartan's Lemma for a fixed $\beta$ to obtain

$$
\Psi_{a}^{\alpha} \in \operatorname{span}\left\{\theta_{j}^{\beta}, \varphi\right\}, \quad a>q .
$$

But $\Psi_{a}^{\alpha}$ is independent of the choice of $\beta$. Therefore, since $\theta_{j}{ }^{\beta}$ are linearly independent and we have assumed $q \geq 2$, we conclude that

$$
\Psi_{a}^{\alpha}=0 \bmod \varphi, \quad a>q
$$

and hence (5.13) implies

$$
\eta_{a \alpha}^{k} \varphi_{\gamma}^{\alpha}=0, \quad a>q
$$

which in view of (5.6) yields

$$
\Theta_{a}^{j}=0, \quad a>q .
$$

5.3. Reducing the freedom for $\Theta_{\alpha}{ }^{J}$ for $J>n$. Next we use (5.11) in case $a=\alpha \leq q$ and $J>n$ that becomes

$$
\theta_{\alpha}^{k} \wedge \Omega_{k}^{J}=\eta_{\alpha}^{J}{ }_{\beta}^{\gamma} \theta_{\gamma}^{k} \wedge \theta_{k}^{\beta} \bmod \varphi, \quad J>n .
$$

Since $\theta_{\alpha}{ }^{k}$ are linearly independent and $(1,0)$, whereas $\theta_{k}^{\beta}$ are $(0,1)$, the term with $\theta_{\gamma}{ }^{k} \wedge \theta_{k}{ }^{\beta}, \gamma \neq \alpha$, in the right-hand side cannot occur in the left-hand side. Therefore

$$
\eta_{\alpha \beta}^{J \gamma}=0 \text { if } \gamma \neq \alpha, \quad J>n,
$$

and hence (5.6) becomes

$$
\Theta_{\alpha}^{J}=\eta_{\alpha \beta}^{J} \varphi_{\alpha}^{\beta}, \quad J>n,
$$

where

$$
\eta_{\alpha \beta}^{J}:=\eta_{\alpha \beta}^{J}{ }^{J},
$$

and (5.16) becomes

$$
\theta_{\alpha}^{k} \wedge \Omega_{k}^{J}=\eta_{\alpha}^{J} \theta_{\alpha}^{k} \wedge \theta_{k}^{\beta} \quad \bmod \varphi, \quad J>n,
$$

i.e.

$$
\theta_{\alpha}^{k} \wedge\left(\Omega_{k}^{J}-\eta_{\alpha \beta}^{J} \theta_{k}^{\beta}\right)=0 \bmod \varphi, \quad J>n .
$$

Then using linear independence of $\theta_{\alpha}{ }^{k}$ and applying Cartan's Lemma, we obtain

$$
\Omega_{k}^{J}=\eta_{\alpha}^{J} \theta_{k}^{\beta} \bmod \left\{\varphi, \theta_{\alpha}\right\}, \quad J>n .
$$

Since $\Omega_{k}^{J}$ is independent of $\alpha$ and $q \geq 2$ by our assumption, taking $(0,1)$ parts we obtain

$$
\eta_{\alpha \beta}^{J}=\eta_{\beta}^{J}, \quad J>n,
$$


for some $\eta_{\beta}^{J}$, hence (5.17) implies

$$
\Theta_{\alpha}^{J}=\eta_{\beta}^{J} \varphi_{\alpha}^{\beta}, \quad J>n,
$$

and (5.20) yields

$$
\Omega_{k}^{J}=\eta_{\beta}^{J} \theta_{k}^{\beta} \bmod \varphi, \quad J>n,
$$

where we dropped $\theta_{\alpha}$ on the right-hand side, since now both sides are independent of $\alpha$ and since $q \geq 2$ by our assumption.

5.4. Reducing the freedom for $\Theta_{\alpha}{ }^{j}$. Here we use the structure equation (5.10) in case $a=\alpha \leq q$ and $b=\beta \leq q$. Then the forms $\varphi_{\alpha}^{\gamma}, \gamma \neq \beta$, and $\varphi_{\delta}^{\beta}, \delta \neq \alpha$, together with $\varphi_{\alpha}^{\beta}$ are linearly independent. Therefore identifying the coefficients in front of these forms in (5.10) and using Cartan's Lemma yields

$$
\begin{aligned}
\Psi_{\alpha}^{\gamma}-\psi_{\alpha}^{\gamma}=0 & \bmod \left\{\theta_{\alpha}, \overline{\theta_{\beta}}, \varphi\right\} \text { if } \gamma \neq \alpha, \\
\left(\Psi_{\alpha}^{\alpha}-\psi_{\alpha}^{\alpha}\right)-\left(\widehat{\Psi}_{\beta}^{\beta}-\widehat{\psi}_{\beta}^{\beta}\right)=0 & \bmod \left\{\theta_{\alpha}, \overline{\theta_{\beta}}, \varphi\right\} .
\end{aligned}
$$

Since $\left(\Psi_{\alpha}^{\gamma}-\psi_{\alpha}^{\gamma}\right), \gamma \neq \alpha$, is independent of the choice of $\beta$ and $q \geq 2$, we conclude

$$
\Psi_{\alpha}^{\gamma}-\psi_{\alpha}^{\gamma}=0 \bmod \left\{\theta_{\alpha}, \varphi\right\} \text { if } \gamma \neq \alpha
$$

Substituting now (5.26) into (5.11) for $a=\alpha \leq q, J=j \leq n$, and identifying coefficients in front of $\theta_{\gamma}{ }^{k} \wedge \theta_{k}{ }^{\beta}$ for $\gamma \neq \alpha$, since $\theta_{\gamma}{ }^{j}$ are $(1,0)$ and linearly independent, whereas $\theta_{j}{ }^{\alpha}=-\overline{\theta_{\alpha}{ }^{j}}$ are $(0,1)$, we obtain

$$
\eta_{\alpha \beta}^{j \gamma}=0 \text { if } \gamma \neq \alpha
$$

In view of (5.26) we can write

$$
\Psi_{\alpha}^{\gamma}-\psi_{\alpha}^{\gamma}=h_{\alpha k}^{\gamma} \theta_{\alpha}^{k} \bmod \varphi, \quad \gamma \neq \alpha
$$

for suitable $h_{\alpha k}^{\gamma}$ and put

$$
h_{\alpha k}^{\alpha}:=0 .
$$

Then (5.11) for $a=\alpha \leq q$ and $J=j \leq n$ becomes

$$
\left\{\left(\Psi_{\alpha}^{\alpha}-\psi_{\alpha}^{\alpha}\right) \delta_{k}^{j}-\left(\Omega_{k}^{j}-\omega_{k}^{j}\right)\right\} \wedge \theta_{\alpha}^{k}+\sum_{\gamma \neq \alpha}\left(\Psi_{\alpha}^{\gamma}-\psi_{\alpha}^{\gamma}\right) \wedge \theta_{\gamma}^{j}=\eta_{\alpha}^{j}{ }_{\gamma} \theta_{\alpha}^{k} \wedge \theta_{k}^{\gamma} \bmod \varphi
$$

where

Substituting (5.27) yields

$$
\eta_{\alpha \beta}^{j}:=\eta_{\alpha \beta}^{j \alpha}
$$

$$
\left\{\left(\Psi_{\alpha}^{\alpha}-\psi_{\alpha}^{\alpha}\right) \delta_{k}^{j}-\left(\Omega_{k}^{j}-\omega_{k}^{j}+h_{\alpha k}^{\gamma} \theta_{\gamma}^{j}-\eta_{\alpha}^{j} \theta_{k}^{\gamma}\right)\right\} \wedge \theta_{\alpha}^{k}=0 \bmod \varphi .
$$

Now using Cartan's Lemma we obtain

$$
\left(\Psi_{\alpha}^{\alpha}-\psi_{\alpha}^{\alpha}\right) \delta_{k}^{j}-\left(\Omega_{k}^{j}-\omega_{k}^{j}\right)=-h_{\alpha k}^{\gamma} \theta_{\gamma}^{j}+\eta_{\alpha \gamma}^{j} \theta_{k}^{\gamma} \bmod \left\{\varphi, \theta_{\alpha}\right\} .
$$

As consequence of (5.25) and (2.8) we also have

$$
\left(\Psi_{\alpha}^{\alpha}-\psi_{\alpha}^{\alpha}\right)+\left(\Psi_{\bar{\beta}}^{\bar{\beta}}-\psi_{\bar{\beta}}^{\bar{\beta}}\right)=0 \bmod \left\{\theta_{\alpha}, \overline{\theta_{\beta}}, \varphi\right\}
$$


and, in particular,

$$
\Re\left(\Psi_{\alpha}^{\alpha}-\psi_{\alpha}^{\alpha}\right)=0 \bmod \left\{\theta_{\alpha}, \overline{\theta_{\alpha}}, \varphi\right\} .
$$

Furthermore, since $\Omega_{j}{ }^{k}$ and $\omega_{j}{ }^{k}$ are antihermitian in view of (2.8), taking hermitian part with respect to $(j, k)$ of $(5.29)$, using (5.30) and identifying coefficients in front of $\theta_{k}^{\gamma}=-\overline{\theta_{\gamma}}{ }^{k}$, we obtain

$$
\overline{h_{\alpha j}^{\gamma}}+\eta_{\alpha \gamma}^{j}=0, \text { if } \gamma \neq \alpha \text {. }
$$

Next, since $\Omega_{j}{ }^{k}$ and $\omega_{j}{ }^{k}$ are antihermitian, (5.30) implies

$$
\left(\Psi_{\alpha}^{\alpha}-\psi_{\alpha}^{\alpha}\right)-\left(\Omega_{j}^{j}-\omega_{j}{ }^{\alpha}\right)=-\left(\Psi_{\bar{\beta}}^{\bar{\beta}}-\psi_{\bar{\beta}}^{\bar{\beta}}\right)+\left(\Omega_{\bar{j}}^{\bar{j}}-\omega_{\bar{j}}^{\bar{j}}\right) \bmod \left\{\varphi, \theta_{\alpha}, \overline{\theta_{\beta}}\right\} .
$$

Hence using (5.29) for $k=j$ and adding its conjugate with $\alpha$ replaced by $\beta$, using (5.32) and identifying the coefficients in front of $\theta_{j}^{\gamma}$ we obtain

$$
\eta_{\alpha \gamma}^{j}=\eta_{\beta \gamma}^{j}, \quad \gamma \neq \beta
$$

and hence

for suitable $\eta_{\gamma}^{j}$. Hence (5.6) implies

$$
\eta_{\alpha \gamma}^{j}=\eta_{\gamma}^{j}
$$

$$
\Theta_{\alpha}^{j}-\theta_{\alpha}^{j}=\eta_{\beta}^{j} \varphi_{\alpha}^{\beta}
$$

5.5. Determination of $\Theta_{a}{ }^{J}$ by a change of frame. Let $\left(B_{a}{ }^{J}\right)$ be a matrix defined by

$$
B_{\alpha}^{J}:=\eta_{\alpha}^{J}, \quad B_{a}^{J}:=0, \quad a>q,
$$

where $\eta_{\alpha}^{J}$ is defined by (5.21) for $J>n$. Consider the change of frame of $S_{p^{\prime}, q^{\prime}}$ discussed after Definition 2.3, given by

$$
\widetilde{Z}_{a}=Z_{a}, \quad \widetilde{X}_{J}=X_{J}+C_{J}^{b} Z_{b}, \quad \widetilde{Y}_{a}=Y_{a}+A_{a}^{b} Z_{b}+B_{a}^{J} X_{J}
$$

such that

$$
C_{J}^{a}:=-B_{J}^{a}
$$

and $A_{a}^{b}$ satisfies

$$
\left(A_{a}^{b}+\overline{A_{b}^{a}}\right)+\sum_{J} B_{a}^{J} \overline{B_{b}^{J}}=0 .
$$

Since the sum here is hermitian, one can always choose $A_{a}^{b}$ with this property. Then $\Phi_{a}^{b}=\varphi_{a}^{b}$ remain the same while $\Theta_{a}^{J}$ change to

$$
\Theta_{a}{ }^{J}-\varphi_{a}^{b} B_{b}^{J}
$$

Therefore (5.33) becomes

$$
\Theta_{a}^{J}=\theta_{a}^{J}
$$

which is equivalent to $\eta_{\beta}^{J}=0$ and hence $\eta_{\alpha}{ }^{j}{ }=0$ and $h_{\alpha}{ }^{\gamma}{ }_{j}=0$ in view of (5.28) and (5.32). Therefore (5.29) implies

$$
\left(\Psi_{\alpha}^{\alpha}-\psi_{\alpha}^{\alpha}\right) \delta_{k}^{j}-\left(\Omega_{k}^{j}-\omega_{k}^{j}\right)=0 \bmod \left\{\varphi, \theta_{\alpha}\right\}
$$


and (5.27) together with (5.14) implies

$$
\Psi_{a}^{\gamma}-\psi_{a}^{\gamma}=0 \bmod \varphi, \text { if } \gamma \neq a,
$$

and, since the left-hand side of (5.35) is independent of $\alpha$ for $j \neq k$, together with (5.23) we obtain

$$
\Omega_{k}^{J}-\omega_{k}^{J}=0 \bmod \varphi \text {, if } J \neq k .
$$

\section{Determination of $\Psi_{a}^{\beta}$ AND $\Omega_{k}{ }^{J}$}

Next, we use (5.34) in the structure equations for $d \theta_{a}{ }^{J}=d \Theta_{a}{ }^{J}$, which yield

$$
\left(\Psi_{a}^{\beta}-\psi_{a}^{\beta}\right) \wedge \theta_{\beta}{ }^{J}+\theta_{a}^{k} \wedge\left(\Omega_{k}^{J}-\omega_{k}^{J}\right)+\varphi_{a}^{\beta} \wedge\left(\Sigma_{\beta}^{J}-\sigma_{\beta}{ }^{J}\right)=0 .
$$

6.1. Determination of $\Psi_{a}^{\beta}$ for $a>q$. In case $a>q, J=j \leq n,(6.1)$ takes the form

$$
\Psi_{a}^{\beta} \wedge \theta_{\beta}^{j}=0, \quad a>q
$$

Together with (5.36), this yields

$$
\Psi_{a}^{\beta}=0, \quad a>q
$$

6.2. Determination of $\Omega_{k}^{J}$ and $\Sigma_{\beta}{ }^{J}$ for $J>n$. Next use (6.1) for $a=\alpha \leq q$ and $J>n$ to obtain

$$
\theta_{\alpha}^{k} \wedge \Omega_{k}^{J}+\varphi_{\alpha}^{\beta} \wedge \Sigma_{\beta}^{J}=0, \quad J>n .
$$

By Cartan's Lemma,

$$
\Omega_{k}^{J}=0 \bmod \left\{\theta_{\alpha}, \varphi_{\alpha}\right\}, \quad \Sigma_{\beta}^{J}=0 \bmod \left\{\theta_{\alpha}, \varphi_{\alpha}\right\}, \quad J>n,
$$

where $\varphi_{\alpha}$ is the ideal generated by $\varphi_{\alpha}^{\beta}$ for $\alpha$ fixed. Since $\Omega_{k}^{J}$ and $\Sigma_{\beta}^{J}$ are independent of $\alpha$ and $q \geq 2$, we conclude

$$
\Omega_{k}^{J}=\Sigma_{\beta}^{J}=0, \quad J>n
$$

We summarize the obtained alignment of the connection forms:

Proposition 6.1. For any local $C R$ embedding $f$ from $S_{p, q}$ into $S_{p^{\prime}, q^{\prime}}$, there is a choice of sections of the frame bundles $\mathcal{B}_{p, q} \rightarrow S_{p, q}$ and $\mathcal{B}_{p^{\prime}, q^{\prime}} \rightarrow S_{p^{\prime}, q^{\prime}}$ such that

$$
\begin{aligned}
& \Phi_{a}{ }^{b}=\varphi_{a}{ }^{b}, \quad \Theta_{a}{ }^{J}=\theta_{a}{ }^{J}, \\
& \Psi_{a}{ }^{\beta}=0, \quad \Omega_{j}{ }^{K}=0, \quad \Sigma_{\alpha}{ }^{K}=0, \quad a>q, \quad K>n .
\end{aligned}
$$

Remark 6.2. Similarly to Remark 4.3, we can restrict to changing only the section of the second bundle $\mathcal{B}_{p^{\prime}, q^{\prime}} \rightarrow S_{p^{\prime}, q^{\prime}}$ 


\section{EmbedDability in A Plane OF SUITABle Dimension}

Proposition 7.1. Under the assumptions of Theorem 1.1, there exist a $(p+q)$-dimensional subspace $V_{1}$ and $a\left(q^{\prime}-q\right)$-dimensional subspace $V_{2}$ in $\mathbb{C}^{p^{\prime}+q^{\prime}}$ with $V_{1} \cap V_{2}=0$ and such that $\langle\cdot, \cdot\rangle$ nondegenerate of signature $(p, q)$ when restricted to $V_{1}$ and null when restricted to $V_{2}$ such that $f\left(S_{p, q}\right) \subset G r\left(V_{1}, q\right) \oplus V_{2}$.

Proof. Denote by $M \subset S_{p, q}$ the open subset where $f$ is defined. Let $Z, X, Y$ be constant vector fields of $\mathbb{C}^{p^{\prime}+q^{\prime}}$ forming a $S_{p^{\prime}, q^{\prime}}$-frame adapted to $M$ at a fixed reference point in $M$. Let

$$
\begin{gathered}
\widetilde{Z}_{a}=\lambda_{a}{ }^{b} Z_{b}+\eta_{a}{ }^{K} X_{K}+\zeta_{a}{ }^{b} Y_{b}, \\
\widetilde{X}_{J}=\lambda_{J}{ }^{b} Z_{b}+\eta_{J}{ }^{K} X_{K}+\zeta_{J}{ }^{b} Y_{b}, \\
\widetilde{Y}_{a}=\tilde{\lambda}_{a}{ }^{b} Z_{b}+\tilde{\eta}_{a}{ }^{K} X_{K}+\tilde{\zeta}_{a}{ }^{b} Y_{b}
\end{gathered}
$$

be an adapted $S_{p^{\prime}, q^{\prime}}$-frame along $M$. Write

$$
A=\left(\begin{array}{ccc}
\lambda_{a}^{b} & \eta_{a}{ }^{K} & \zeta_{a}^{b} \\
\lambda_{J}^{b} & \eta_{J}{ }^{K} & \zeta_{J}^{b} \\
\tilde{\lambda}_{a}^{b} & \tilde{\eta}_{a}{ }^{K} & \tilde{\zeta}_{a}^{b}
\end{array}\right),
$$

so that (7.1) - (7.3) take the form

$$
\left(\begin{array}{l}
\widetilde{Z} \\
\widetilde{X} \\
\widetilde{Y}
\end{array}\right)=A\left(\begin{array}{l}
Z \\
X \\
Y
\end{array}\right)
$$

Since $Z, X, Y$ form an adapted frame at a reference point of $M$, we may assume that

$$
A=I_{p^{\prime}+q^{\prime}}
$$

at the reference point. Since $Z, X, Y$ are constant vector fields, i.e., $d Z=d X=d Y=0$, differentiating (7.5) and using (2.5) we obtain

$$
d A=\Pi A
$$

where $\Pi$ is the connection matrix of $S_{p^{\prime}, q^{\prime}}$, i.e.

$$
d A=\left(\begin{array}{ccc}
\Psi_{a}^{b} & \Theta_{a}^{J} & \Phi_{a}^{b} \\
\Sigma_{K}^{b} & \Omega_{K}^{J} & \Theta_{K}^{b} \\
\Xi_{a}^{b} & \Sigma_{a}^{J} & \widehat{\Psi}_{a}^{b}
\end{array}\right) A
$$

Next, it follows from Proposition 6.1 that

$$
d \widetilde{Z}_{a}=\sum_{b>q} \Psi_{a}^{b} \widetilde{Z}_{b}, \quad a>q
$$

in particular, the span of $\widetilde{Z}_{a}, a>q$, is independent of the point in $M$. Hence together with (7.1) and (7.6), we conclude

$$
\eta_{a}{ }^{K}=\zeta_{a}{ }^{b}=0, \quad a>q
$$


Furthermore, (7.8) implies

$$
\left(\begin{array}{l}
d \eta_{a}{ }^{K} \\
d \eta_{J}^{K} \\
d \tilde{\eta}_{a}^{K}
\end{array}\right)=\left(\begin{array}{ccc}
\Psi_{a}^{b} & \Theta_{a}{ }^{L} & \Phi_{a}^{b} \\
\Sigma_{J}^{b} & \Omega_{J}{ }^{L} & \Theta_{J}^{b} \\
\Xi_{a}^{b} & \Sigma_{a}{ }^{L} & \widehat{\Psi}_{a}^{b}
\end{array}\right)\left(\begin{array}{l}
\eta_{b}{ }^{K} \\
\eta_{L}{ }^{K} \\
\tilde{\eta}_{b}{ }^{K}
\end{array}\right)
$$

In particular, restricting to $a=\alpha \leq q$ and $J=j \leq n$ and using Proposition 6.1 (together with the symmetry relations analogous to (2.8)) we obtain

$$
\left(\begin{array}{l}
d \eta_{\alpha}{ }^{K} \\
d \eta_{j}{ }^{K} \\
d \tilde{\eta}_{\alpha}{ }^{K}
\end{array}\right)=\left(\begin{array}{ccc}
\Psi_{\alpha}{ }^{b} & \theta_{\alpha}{ }^{L} & \varphi_{\alpha}{ }^{b} \\
\Sigma_{j}{ }^{b} & \Omega_{j}{ }^{L} & \theta_{j}{ }^{b} \\
\Xi_{\alpha}{ }^{b} & \Sigma_{\alpha}{ }^{L} & \widehat{\Psi}_{\alpha}{ }^{b}
\end{array}\right)\left(\begin{array}{l}
\eta_{b}{ }^{K} \\
\eta_{L}{ }^{K} \\
\tilde{\eta}_{b}{ }^{K}
\end{array}\right) .
$$

Now with (7.10) and Proposition 6.1 taken into account, (7.12) becomes

$$
\left(\begin{array}{l}
d \eta_{\alpha}{ }^{K} \\
d \eta_{j}{ }^{K} \\
d \tilde{\eta}_{\alpha}{ }^{K}
\end{array}\right)=\left(\begin{array}{ccc}
\Psi_{\alpha}{ }^{\beta} & \theta_{\alpha}{ }^{l} & \varphi_{\alpha}{ }^{\beta} \\
\Sigma_{j}{ }^{\beta} & \Omega_{j}{ }^{l} & \theta_{j}{ }^{\beta} \\
\Xi_{\alpha}{ }^{\beta} & \Sigma_{\alpha}{ }^{l} & \widehat{\Psi}_{\alpha}{ }^{\beta}
\end{array}\right)\left(\begin{array}{l}
\eta_{\beta}{ }^{K} \\
\eta_{l}{ }^{K} \\
\tilde{\eta}_{\beta}{ }^{K}
\end{array}\right) .
$$

Repeating the above argument for $\zeta$ instead of $\eta$, we obtain

$$
\left(\begin{array}{c}
d \zeta_{\alpha}{ }^{b} \\
d \zeta_{j}^{b} \\
d \widetilde{\zeta}_{\alpha}^{b}
\end{array}\right)=\left(\begin{array}{ccc}
\Psi_{\alpha}{ }^{\beta} & \theta_{\alpha}{ }^{l} & \varphi_{\alpha}{ }^{\beta} \\
\Sigma_{j}{ }^{\beta} & \Omega_{j}{ }^{l} & \theta_{j}{ }^{\beta} \\
\Xi_{\alpha}{ }^{\beta} & \Sigma_{\alpha}{ }^{l} & \widehat{\Psi}_{\alpha}^{\beta}
\end{array}\right)\left(\begin{array}{c}
\zeta_{\beta}^{b} \\
\zeta_{l}^{b} \\
\widetilde{\zeta}_{\beta}^{b}
\end{array}\right)
$$

Thus each of the vector valued functions $\eta^{K}:=\left(\eta_{\alpha}{ }^{K}, \eta_{j}{ }^{K}, \tilde{\eta}_{\alpha}{ }^{K}\right)$ for a fixed $K$ and $\zeta^{b}:=\left(\zeta_{\alpha}{ }^{b}, \zeta_{j}{ }^{b}, \tilde{\zeta}_{\alpha}{ }^{b}\right)$ for a fixed $b$ satisfies a complete system of linear first order differential equations. Then by the initial condition (7.6) and the uniqueness of solutions, we conclude, in particular, that

$$
\eta^{K}=\zeta^{b}=0, \quad K>n, b>q .
$$

Hence (7.5) implies

$$
\widetilde{Z}_{\alpha}=\lambda_{\alpha}^{b} Z_{b}+\eta_{\alpha}^{k} X_{k}+\zeta_{\alpha}^{\beta} Y_{\beta}
$$

Now setting

$$
\widehat{Z}_{\alpha}:=\widetilde{Z}_{\alpha}-\sum_{b>q} \lambda_{\alpha}^{b} Z_{b}
$$

we still have

$$
\operatorname{span}\left\{\widehat{Z}_{\alpha}, \widetilde{Z}_{q+1}, \ldots, \widetilde{Z}_{q^{\prime}}\right\}=\operatorname{span}\left\{\widetilde{Z}_{a}\right\}
$$

whereas (7.16) becomes

$$
\widehat{Z}_{\alpha}=\lambda_{\alpha}^{\beta} Z_{\beta}+\eta_{\alpha}{ }^{k} X_{k}+\zeta_{\alpha}^{\beta} Y_{\beta},
$$

implying

$$
\operatorname{span}\left\{\widehat{Z}_{\alpha}\right\} \subset \operatorname{span}\left\{Z_{1}, \ldots, Z_{q}, X_{1}, \ldots, X_{n}, Y_{1}, \ldots, Y_{q}\right\} \text {. }
$$

Then together with (7.9) we conclude that

$$
f(M)=\operatorname{span}\left\{\widetilde{Z}_{a}\right\}=\operatorname{span}\left\{\widehat{Z}_{\alpha}\right\} \oplus \operatorname{span}\left\{\widetilde{Z}_{q+1}, \ldots, \widetilde{Z}_{q^{\prime}}\right\}
$$


where

$$
=\operatorname{span}\left\{\widehat{Z}_{\alpha}\right\} \oplus \operatorname{span}\left\{Z_{q+1}, \ldots, Z_{q^{\prime}}\right\} \subset G r\left(V_{1}, q\right) \oplus V_{2}
$$

$$
V_{1}=\operatorname{span}\left\{Z_{1}, \ldots, Z_{q}, X_{1}, \ldots, X_{n}, Y_{1}, \ldots, Y_{q}\right\}, \quad V_{2}=\operatorname{span}\left\{Z_{q+1}, \ldots, Z_{q^{\prime}}\right\}
$$

\section{Rigidity of CR Embeddings From $S_{p, q}$ TO $S_{p^{\prime}, q^{\prime}}$}

As consequence of Proposition 7.1, we conclude that, after a linear change of coordinates, $f(M)$ locally coincides with $S_{p, q}$ linearly embedded into $S_{p^{\prime}, q^{\prime}}$. Identifying $M=S_{p, q}$ with its image, $f$ becomes a local CR-automorphism of $M$. Then by a theorem of Kaup-Zaitsev [KZ06, Theorem 4.5], $f$ is a restriction of a global CR-automorphism of $S_{p, q}$. Furthermore, by [KZ00, Theorem 8.5], $f$ extends to a biholomorphic automorphism of the bounded symmetric domain and the rigidity follows.

\section{REFERENCES}

[A74] Alexander, H. - Holomorphic mappings from the ball and polydisc, Math. Ann. 209 (1974), 249-256.

[BEH08] Baouendi, M. S.; Ebenfelt, P.; Huang, X. - Super-rigidity for CR embeddings of real hypersurfaces into hyperquadrics. Adv. Math. 219 (2008), no. 5, 1427-1445.

[BEH09] Baouendi, M. S.; Ebenfelt, P.; Huang, X. - Holomorphic Mappings between Hyperquadrics with Small Signature Difference. American J. Math. 133 (2011), no. 6, 1633-1661.

[BH05] Baouendi, M. S.; Huang, X. - Super-rigidity for holomorphic mappings between hyperquadrics with positive signature. J. Differential Geom. 69 (2005), no. 2, 379-398.

[BER99] Baouendi, M.S.; Ebenfelt, P.; Rothschild, L.P. - Real Submanifolds in Complex Space and Their Mappings. Princeton Math. Series 47, Princeton Univ. Press, 1999.

[Bo47] Bochner, S. Curvature in Hermitian metric. Bull. Amer. Math. Soc. 53 (1947), 179-195.

[Ca53] Calabi, E. - Isometric imbedding of complex manifolds. Ann. of Math. (2) 58 (1953), 1-23.

[CT93] Carlson, J. A.; Toledo, D. - Rigidity of harmonic maps of maximum rank. J. Geom. Anal. 3 (1993), no. 2, 99-140.

[CM74] Chern, S.S; Moser, J.K. - Real hypersurfaces in complex manifolds. Acta Math. 133 (1974), 219-271.

[CS83] Cima, J.; Suffridge, T. J. - A reflection principle with applications to proper holomorphic mappings. Math. Ann. 265 (1983), 489-500.

[CS90] Cima, J.; Suffridge, T. J. - Boundar behavior of rational proper maps. Duke Math. 60 (1990), 135-138.

[EHZ04] Ebenfelt, P.; Huang, X.; Zaitsev, D. - Rigidity of CR-immersions into spheres. Comm. in Analysis and Geometry $\mathbf{1 2}$ (2004), no. 3, 631-670.

[EHZ05] Ebenfelt, P.; Huang, X.; Zaitsev, D. - The equivalence problem and rigidity for hypersurfaces embedded into hyperquadrics. Amer. J. Math. 127 (2005), no. 1, 169-191.

[ES10] Ebenfelt, P.; Shroff, R. - Partial Rigidity of CR Embeddings of Real Hypersurfaces into Hyperquadrics with Small Signature Difference. http://arxiv.org/abs/1011.1034

[Fa86] Faran V, J. J. - On the linearity of proper maps between balls in the lower codimensional case. $J$. Differential Geom. 24, (1986), 15-17.

[F86] Forstnerič, F. - Proper holomorphic maps between balls. Duke Math. J. 53 (1986), 427-440.

[F89] Forstnerič, F. - Extending proper holomorphic mappings of positive codimension. Invent. Math. 95 (1989), $31-61$.

[H99] Huang, X. - On a linearity problem for proper holomorphic maps between balls in complex spaces of different dimensions. J. Differential Geom. 51 (1999), 13-33.

[HJ01] Huang, X.; Ji, S. - Mapping $B^{n}$ into $B^{2 n-1}$. Invent. Math. 145 (2) (2001), 219-250.

[H03] Huang, X. - On a semi-rigidity property for holomorphic maps. Asian J. Math. 7 (2003), no. 4, 463-492. 
[HJX06] Huang, X.; Ji, S.; Xu, D. - A new gap phenomenon for proper holomorphic mappings from $B^{n}$ into $B^{N}$. Math. Res. Lett. 13 (4) (2006), 515-529.

[HJX07] Huang, X.; Ji, S.; Xu, D. - On some rigidity problems in Cauchy-Riemann analysis. Proceedings of the International Conference on Complex Geometry and Related Fields, 89 - 107, AMS/IP Stud. Adv. Math., 39, Amer. Math. Soc., Providence, RI, 2007.

[JY99] Jost, J.; Yau, S.-T. - Harmonic maps and rigidity theorems for spaces of nonpositive curvature. Comm. Anal. Geom. 7 (1999), no. 4, 681-694.

[KZ00] Kaup, W.; Zaitsev, D. - On symmetric Cauchy-Riemann manifolds. Adv. Math., 149 (2000), no. 2, $145-181$.

[KZ03] Kaup, W.; Zaitsev, D. - On the CR-structure of compact group orbits associated with bounded symmetric domains. Inventiones mathematicae 153 (2003), no. 1, 45-104.

[KZ06] Kaup, W.; Zaitsev, D. - On local CR-transformations of Levi-degenerate group orbits in compact Hermitian symmetric spaces. J. Eur. Math. Soc. 8 (2006), 465-490.

[M89] Mok, N. - Metric Rigidity Theorems on Hermitian Locally Symmetric Spaces. Series in Pure Math., Vol. 6, World Scientific, Singapore, 1989.

[M08] Mok, N. - Nonexistence of proper holomorphic maps between certain classical bounded symmetric domains. Chin. Ann. Math. Ser. B 29 (2008), no. 2, 135-146.

[M11] Mok, N. - Geometry of holomorphic isometries and related maps between bounded domains, in Geometry and Analysis, Vol. II, ALM 18, Higher Education Press and International Press, Beijing-Boston 2011, 225270.

[P07] Poincaré, H. - Les fonctions analytiques de deux variables et la representation conforme, Rend. Circ. Mat. Palermo (2) 23 (1907), 185-220.

[S80] Siu, Y.-T. - The complex-analyticity of harmonic maps and the strong rigidity of compact Kähler manifolds. Ann. of Math. (2) 112 (1980), no. 1, 73-111.

[S81] Siu, Y.-T. - Strong rigidity of compact quotients of exceptional bounded symmetric domains, Duke Math. J. 48 (1981), 857-871.

[Ta62] Tanaka, N. - On the pseudo-conformal geometry of hupersurfaces of the space of $n$ complex variables. $J$. Math. Soc. Japan 14 (1962), 397-429.

[To99] Toledo, D. - Rigidity theorems in Khler geometry and fundamental groups of varieties. Several complex variables (Berkeley, CA, 19951996), 509533, Math. Sci. Res. Inst. Publ., 37, Cambridge Univ. Press, Cambridge, 1999.

[Ts93] Tsai, I-H. - Rigidity of proper holomorphic maps between symmetric domains. J. Differential Geom. 37 (1993), no. 1, 123-160.

[Tu02b] Tu, Z. - Rigidity of proper holomorphic mappings between nonequidimensional bounded symmetric domains. Math. Z. 240 (2002), no. 1, 13-35.

[Tu02a] Tu, Z. - Rigidity of proper holomorphic mappings between equidimensional bounded symmetric domains. Proc. Amer. Math. Soc. 130 (2002), no. 4, $1035-1042$.

[W79] Webster, S. M. - The rigidity of C-R hypersurfaces in a sphere. Indiana Univ. Math. J. 28 (1979), 405-416.

[Y11] Yau, S. S.-T. - Rigidity of CR morphisms between compact strongly pseudoconvex CR manifolds. J. Eur. Math. Soc. 13 (2011), 175-184.

[YZ10] Yuan, Y.; Zhang, Y. - Rigidity for local holomorphic isometric embeddings from $B^{n}$ into $B^{N_{1}} \times \ldots \times B^{N_{m}}$ up to conformal factors. J. Differential Geom. 90 (2012), no.2, 329-349.

S.-Y. Kim: Department of Mathematics Education, Kangwon National University, 123 HyojaDong, Chuncheon, Kangwon-do, 200-701, Korea

E-mail address: sykim87@kangwon.ac.kr

D. Zaitsev: School of Mathematics, Trinity College Dublin, Dublin 2, Ireland

E-mail address: zaitsev@maths.tcd.ie 